\title{
Aging and degeneration of the human macula. 1. Outer nuclear layer and photoreceptors
}

\author{
SAMUEL GARTNER AND PAUL HENKIND \\ From the Montefiore Hospital and Medical Center, Albert Einstein College of Medicine, \\ New York, USA
}

SUMMARY In a light microscopic study of the macula of 104 human eyes obtained at necropsy of patients aged 3 to 96 we found: (1) Displacement of nuclei from the outer nuclear layer into the outer plexiform layer occurred in small numbers early in life and markedly increased after age 30 . (2) Displacement of nuclei from the outer nuclear layer to the layer of rods and cones was rare in early life but increased considerably after age 40. (3) Displacement of nuclei is probably secondary to shrinkage of their attached fibres and is associated with aging. (4) Displaced nuclei apparently undergo changes in size, shape, and chromatin content and may go on to necrosis. (5) Twenty-four of the 104 eyes studied had an obvious reduction in the numbers of nuclei in the outer nuclear layer and their photoreceptors in the macular zone. All were in eyes from patients over age 40 . No concomitant defect was found in the subjacent pigment epithelium, Bruch's membrane, or the choriocapillaris. The loss of nuclei of the outer nuclear layer appears to be a primary retinal disorder associated with aging.

We found in the macula a loss of nuclei from the outer nuclear layer (ONL) which increased with age. This loss apparently starts with displacement or migration of nuclei from the ONL into the outer plexiform layer (OPL) and into the layer of rods and cones. This observation has received little or no attention and we could not find any previous systematic study in the literature.

\section{Material and methods}

One-hundred-and-four eyes obtained at necropsy from patients aged 3 to 96 were selected for study because sections included the macular region. Fiftytwo of these eyes had sections through the fovea. Only eyes without significant inflammatory or degenerative disease or trauma were studied. Eyes with disciform degeneration or with pigment migration into the retina were excluded. The eyes were opened in the horizontal meridian so that sections included the disc and macula. They were prepared with celloidin embedding cut $14 \mathrm{um}$ thick and stained with haematoxylin and eosin. The macula includes the

Correspondence to Professor Paul Henkind, Montefiore Hospital and Medical Center, Albert Einstein College of Medicine, 111 East 210 Street, Bronx, New York 10467, USA. fovea and parafoveal zone. Its outer boundary was the point where the ganglion cell layer was less than 2 cells thick. Sections were examined by light microscopy and the total numbers of displaced nuclei in the macula counted for each section. At least 3 slides of each eye were examined and the results averaged. The data were graphed (Figs. 1 and 2).

\section{Results}

The normal nuclei in the ONL appear uniform with a circular or slightly ovoid shape and contain many fine chromatin granules. Several layers of cone nuclei are concentrated at the fovea. Elsewhere in the macula cone nuclei are near the external limiting membrane and appear slightly larger and paler than the rod nuclei.

The number of nuclei in the ONL does not remain constant during a long lifetime. Significant changes are found with aging. Some nuclei from the ONL become displaced into the OPL (Fig. 1) and others into the layer of rods and cones (Fig. 2).

Displacement of nuclei into the OPL at the macula is evident even early in life but increases after age 30, and is most pronounced after age 50 (Fig. 1). The nuclei displaced into the OPL appear to be mainly rod nuclei from the ONL and perhaps 
Fig. 1 Nuclei displaced into outer plexiform layer.
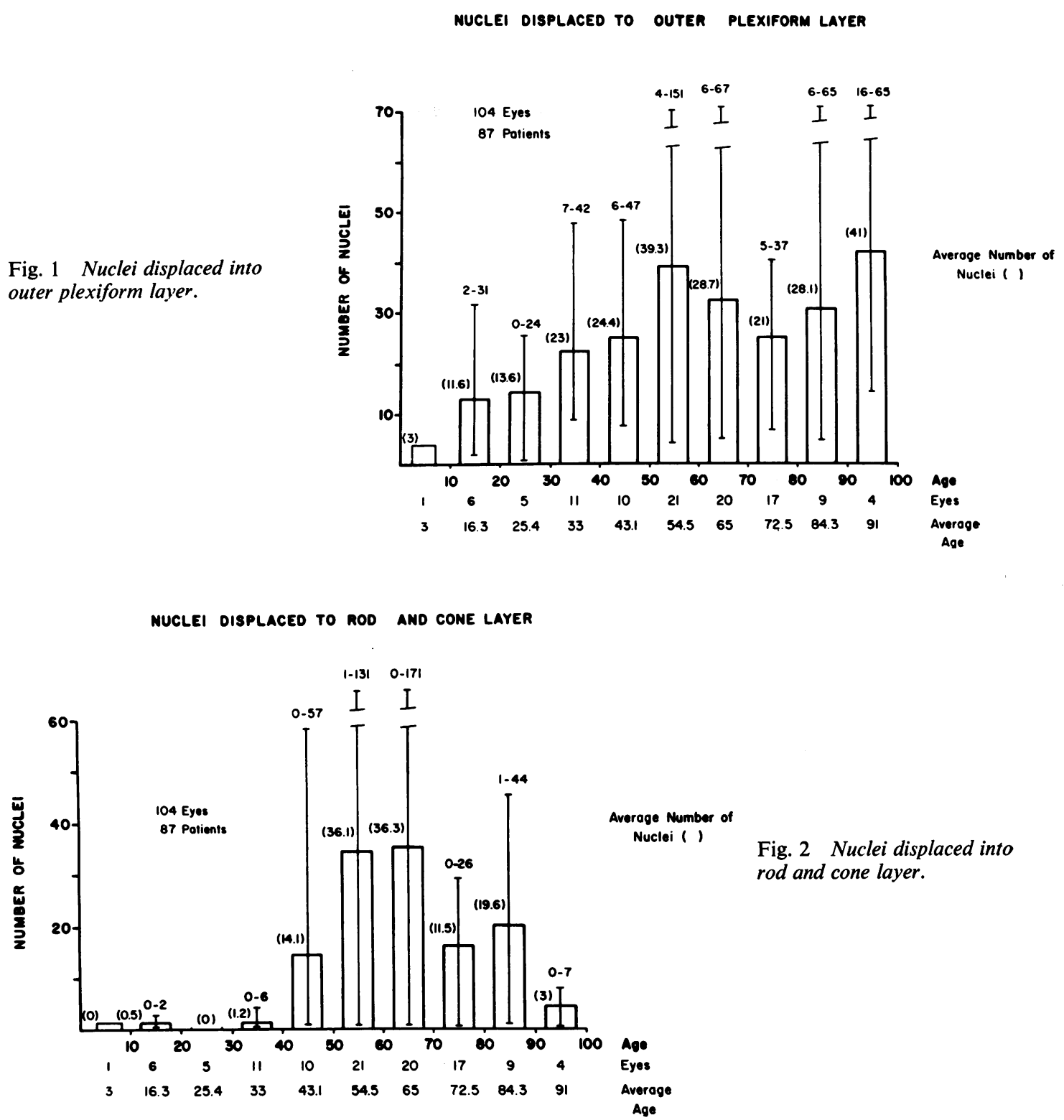

Fig. 2 Nuclei displaced into rod and cone layer.

some nuclei from the inner nuclear layer (INL) (Fig. 3).

Displacement of nuclei from the ONL through the external limiting membrane to the rod and cone layer has been described as a normal variant observed most often in the macula. ${ }^{1}$ However, such displacement is rare in youth (Fig. 2). Only after age 40 is it frequent, being most common after age 50 . A decrease in such nuclei was noted after age 70 . Nuclear displacement is rare in the centre of the 
very irregular, and a few seemed necrotic. In several instances we observed similar degeneration of nuclei within the ONL.

Twenty-four of the 104 eyes studied had discrete areas in the macula with an appreciable loss of nuclei in the ONL. The loss was extensive in 9 and moderate in 15 eyes (Table 1 and Figs. 5 and 6). The loss was usually in small focal areas, but some eyes had a broad zone with a diminished number of nuclei. The loss of nuclei was usually found opposite normal or only slightly defective retinal pigment

Fig. 3 Male age 63. Parafoveal zone. There is a large number of displaced nuclei in the OPL. Most of these nuclei are pale, deformed and elongated and lie parallel to the obliquity of the fibres in Henle's layer. Some nuclei in the ONL are elongated and deformed. Some of the photoreceptors have lost part or all of their outer segments. $(H$ and $E, \times 15)$.

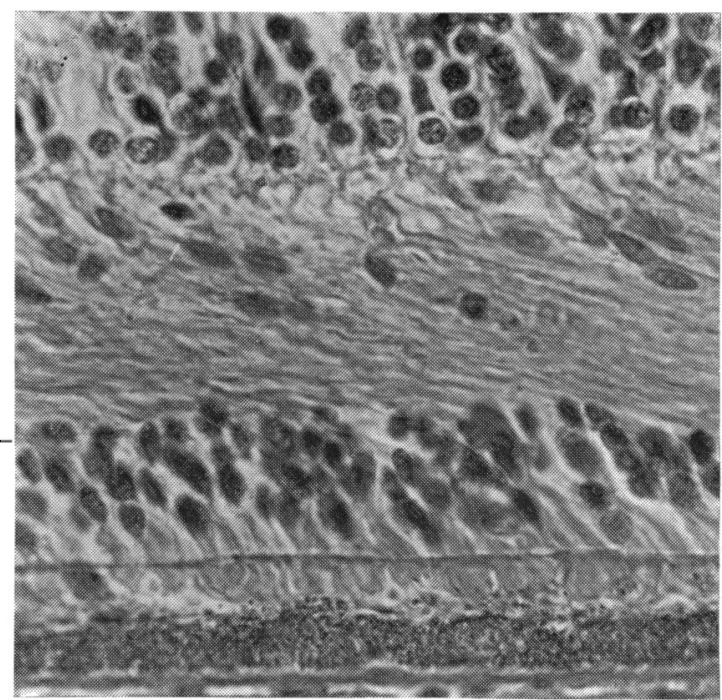

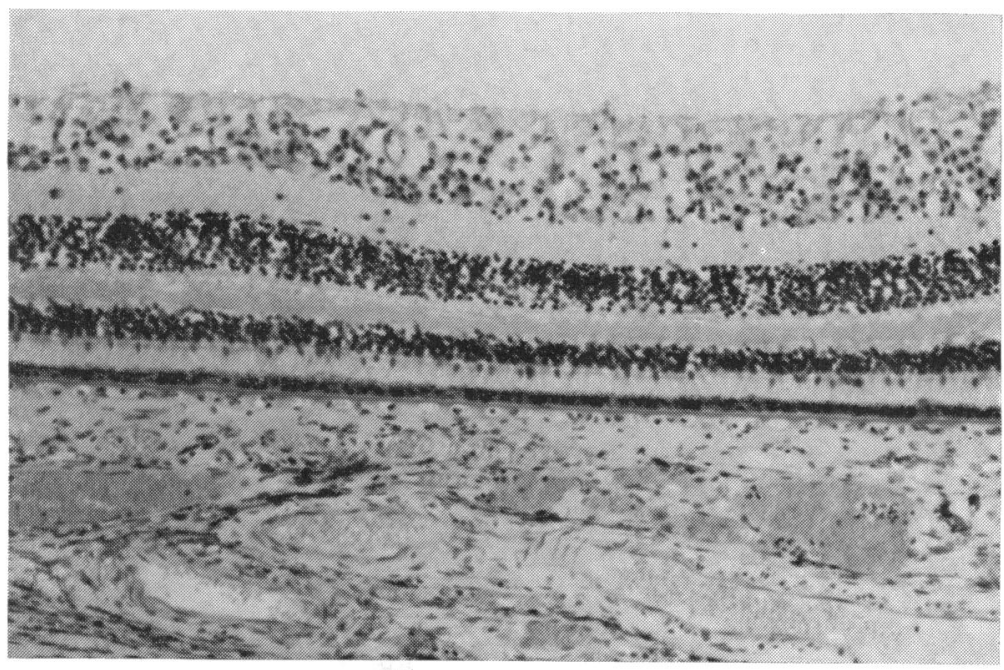

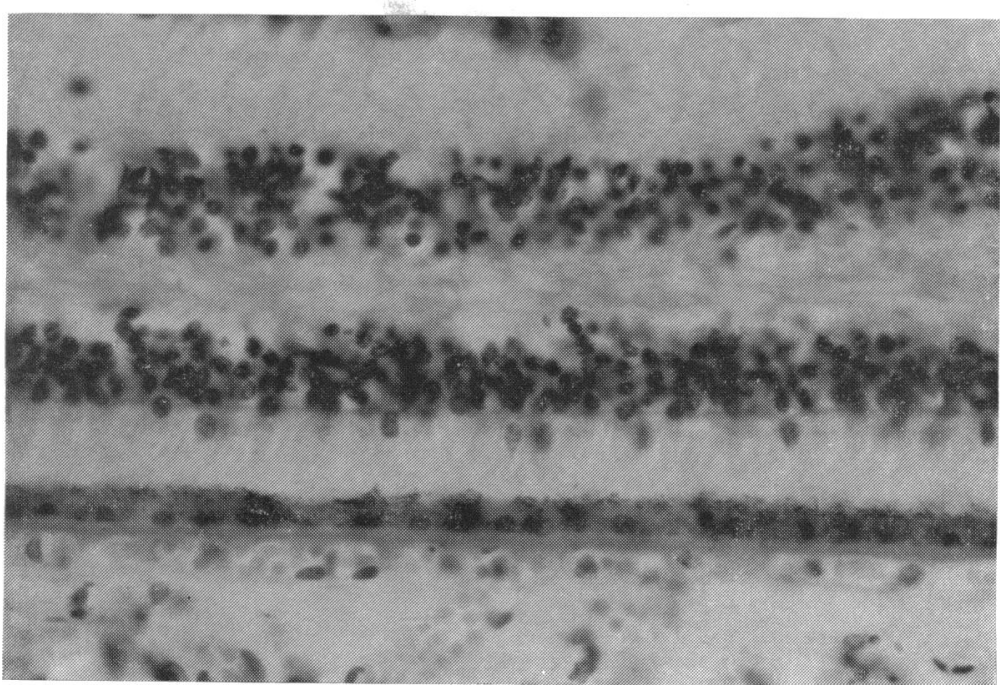


Fig. 5 upper, right eye; lower, left eye. Female aged 41. Parafoveal zone. The ONL is markedly thinned and centrally is only 1 cell thick. The OPL is also narrowed. Many photoreceptors are deformed and shortened with a loss of outer segments. There is a mild degree of irregularity of the retinal pigment epithelium with slight depigmentation. Bruch's membrane and the choriocapillaris appear normal. In Fig. $5 b$ there are numerous displaced nuclei in the rod and cone layer. ( $H$ and $E, \times 15$ ).
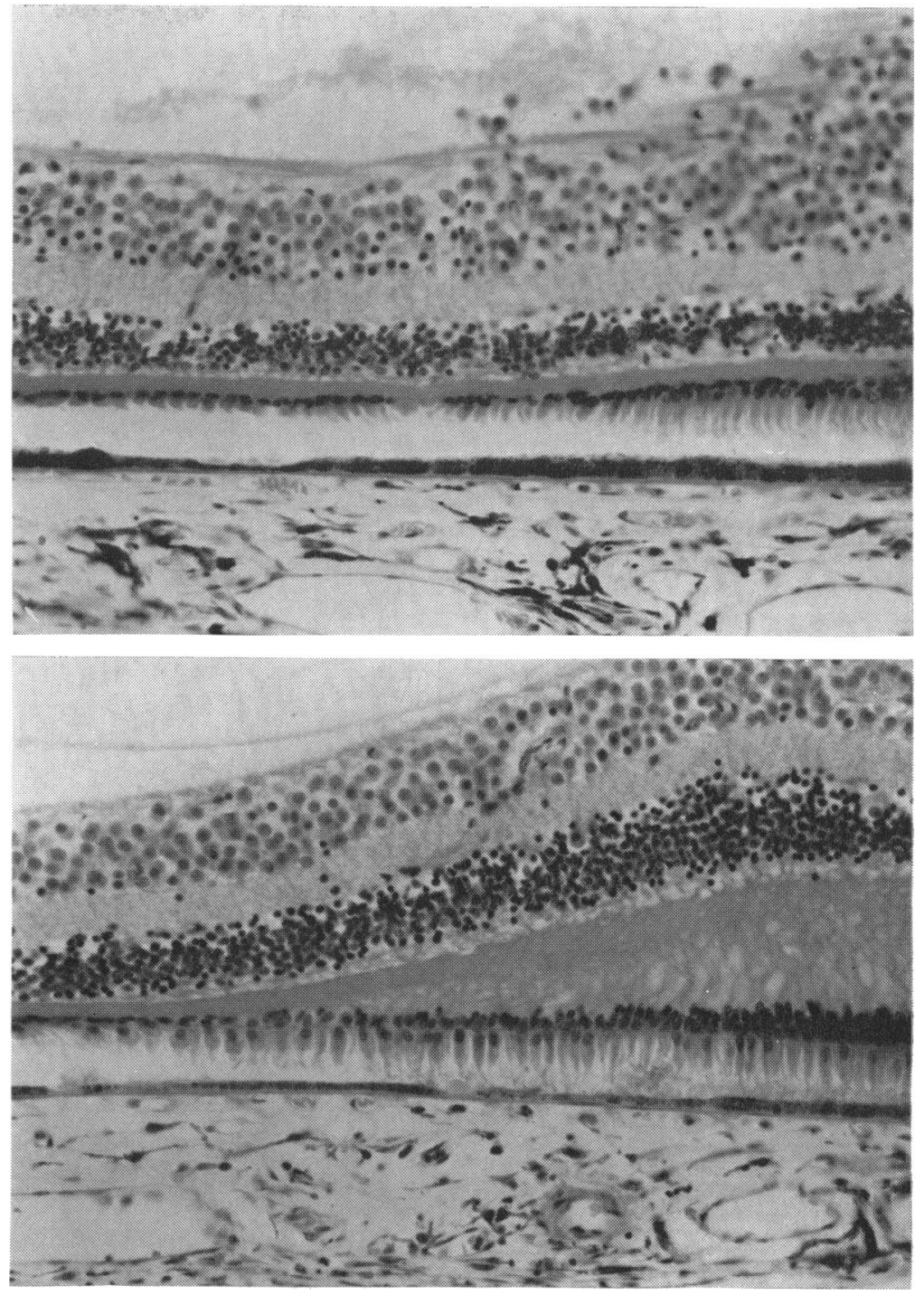

epithelium, Bruch's membrane, and choriocapillaris.

Accompanying the loss of nuclei in the ONL we noted shrunken and deformed rods and cones and a diminution in the numbers of photoreceptors (Figs. 5 and 6). The reduced population of cells in the ONL resulted in a loss of the axons traversing the outer plexiform layer, and that layer appeared thinner than normal (Fig. 4).

Three of the 24 eyes with marked loss of cells from the ONL had interesting fellow eyes. In a patient aged 85 there was typical senile pigmentary degeneration of the macula in the other eye, and 2 patients, aged 71 and 94, had disciform degeneration of the macula in the second eye.
Table 1 Loss of nuclei in outer layer in 104 eyes

\begin{tabular}{lllll}
\hline Age & $\begin{array}{l}\text { Numbers } \\
\text { of } \text { eyes }\end{array}$ & $\begin{array}{l}\text { Marked } \\
\text { loss of } \\
\text { nuclei }\end{array}$ & $\begin{array}{l}\text { Moderate } \\
\text { loss of } \\
\text { nuclei }\end{array}$ & $\begin{array}{l}\% \\
\text { Affected }\end{array}$ \\
\hline Before age 40 & 23 & 0 & 0 & 0 \\
$40-49$ & 10 & 3 & 2 & 50 \\
$50-59$ & 21 & 0 & 7 & $33 \frac{1}{3}$ \\
$60-69$ & 20 & 2 & 2 & 20 \\
$70-79$ & 17 & 2 & 2 & 22 \\
$80-89$ & 9 & 1 & 2 & $33 \frac{1}{3}$ \\
$90-100$ & 4 & 1 & 0 & 25 \\
Total & 104 & 9 & 15 & \\
\hline
\end{tabular}



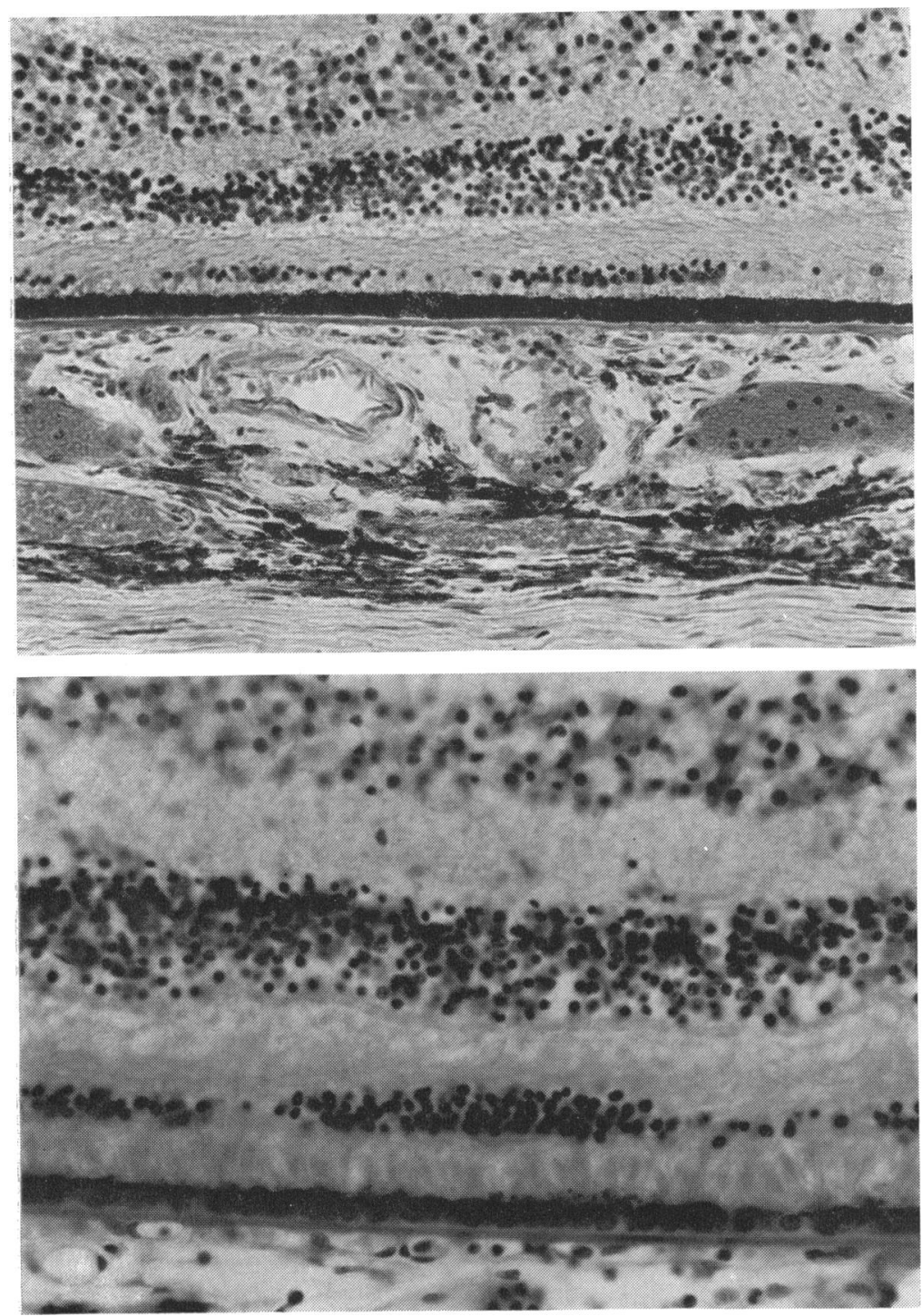

Fig. 6 upper, male aged 62 ; lower, male aged $67 . \quad$ There is irregular loss of nuclei in the ONL as well as of their photoreceptors. The retinal pigment epithelium, Bruch's membrane, and choriocapillaris appear intact.

\section{Discussion}

In 24 of 104 necropsy eyes without significant ocular disease we found a reduced population of nuclei in the ONL along with a loss of their photoreceptors. These observations of human eyes parallel the recent finding that a progressive loss of cells occurs with aging in the ONL in healthy rats. ${ }^{2}$

A loss of retinal neurons and photoreceptors at the macula has been demonstrated by many authors in eyes that had pigmentary migration into a degenerated retina or a disciform response..$^{3-9}$ Those who have studied senile macular degeneration have been impressed with the associated changes in the layers adjacent to the retina, and they have picked one or another layer to assign as the primary site with macular degeneration as a secondary consequence. There is no question that with aging a variety of changes occur in the retinal pigment epithelium, Bruch's membrane, and the choroidal vasculature. These structures are all so closely interrelated that it has been difficult to ascertain the site of a primary defect. However, the present study indicates that a progressive degeneration of cells in the ONL and their photoreceptors may occur without significant changes in the adjacent layers.

The elongation of nuclei found in the rod and cone layer has been explained as a consequence of com- 
pression by the adjacent photoreceptors. ${ }^{10}$ Compression would not, however, explain the change in shape of the nuclei displaced into the OPL. That layer of fibres (Henle's layer) in the macular zone is easily separated by fluid in cystoid oedema and by exudates in diabetes.

What is the explanation for nuclear displacement from the ONL? The nuclei in the ONL are attached at one end to the photoreceptors and on the other to their axonal fibres, which cross the OPL and join with dendrites from the INL. We speculate that nuclear displacement in either direction may be due to traction exerted by shrinkage of the attached fibres. A traction phenomenon could explain elongation of the displaced nuclei in the direction of the nerve fibres in the OPL as well as in the layer of rods and cones. Less likely is the possibility of active nuclear migration.

It is noteworthy that Marshall et al.11 have demonstrated by electron microscopy a change with aging in human rods. Starting at age 40 some rods become elongated and convoluted, and by the seventh decade 10 to $20 \%$ of the rods are affected. The degenerative changes we found also appear after the fourth decade.

A normal retina has about 130000000 nuclei in the ONL with a corresponding number of photoreceptors. ${ }^{12}$ In our material we could not accurately count the changes in numbers of nuclei with aging. Our report is thus qualitative and notes only a substantial loss that is obvious by light microscopy. We found that an appreciable number of nuclei were displaced from the ONL after age 30. Some had undergone degeneration characterised by a change in their size and shape as well as the density of chromatin; in some only a cellular ghost remained. Nuclear displacement probably accounts for the loss of cells in the ONL. In our study we found no correlation between the loss of nuclei in the ONL and changes in the pigment epithelium, Bruch's membrane, or the choriocapillaris. It appears that the cellular diminution in the ONL can be primary and is most likely an aging phenomenon. Along with the loss of cells in the ONL there was similar reduction in number of the attached rods and cones and the axons in the outer plexiform layer. The relation of this process to senile macular degeneration is unclear and requires further investigation.

The loss of nuclei in the ONL and their photoreceptors is likely to cause some change in the visual properties of the eye. It is well known that there are parameters of visual function which diminish with aging without obvious clinical alterations in the lens or the fundus. Our findings may help to explain some of this loss of function.

This work was supported in part by an unrestricted grant from Research to Prevent Blindness Inc.

\section{References}

1 Hogan MJ, Zimmerman LE. Ophthalmic Pathology. Philadelphia: Saunders, 1962: 471.

2 Glatt HJ, Henkind P, Levine H. Aging changes in the retina of the rat. $A R V O$ abstracts no. 12. 1979;82.

3 Junius P, Kuhnt D. Die scheibenformige Entartung der Netzhautmitte: Degeneratio maculae Tutea disciformis. Berlin: Karger, 1926.

4 Klien BA. Some aspects of classification and differential diagnosis of senile macular degeneration. Am J Ophthalmol 1964; 58: 927-39.

5 Kornzweig AL. Diseases of the macula in the aged. Trans Am Acad Ophthalmol Otolaryngol 1965; 69: 66882.

6 Gass JDM. Pathogenesis of disciform detachment of the neuroepithelium. Am J Ophthalmol 1967; 63: 573-85; $587-615 ; 617-45 ; 645-59 ; 661-89 ; 689-711$.

7 Hogan MJ. Bruch's membrane and diseases of the macula. Trans Ophthalmol Soc UK 1968; 87: 113-61.

8 Sarks SH. Ageing and degeneration in the macula region. Br J Ophthalmol 1976; 60: 324-41.

9 Green WR, Key SN. Senile macular degeneration. Trans Am Ophthalmol Soc 1977; 75: 180-254.

10 Polyak SD. The Retina. Chicago: University of Chicago Press, 1941: 204.

11 Marshall JM, Grindle J, Ansell PL, Borwein B. Convolution in human rods. Br J Ophthalmol 1979; 63: 181-7.

12 Østerberg, G. Topography of the layer of rods and cones in the human retina. Acta Ophthalmol (Kbh) 1935; Supplement $6: 1-103$. 